\title{
Preliminary Statistical Analysis of Lambda Bootis Stars
}

\author{
S. Ghazaryan ${ }^{* 1}$, G.Alecian ${ }^{2}$, E.Paunzen ${ }^{3}$, and A.A. Hakobyan ${ }^{4}$ \\ ${ }^{1}$ Byurakan Astrophysical Observatory, 0213 Byurakan, Aragatsotn province, Armenia \\ ${ }^{2}$ LUTH, CNRS, Observatoire de Paris, PSL University, Universite Paris Diderot, Meudon, France \\ ${ }^{3}$ Department of Theoretical Physics and Astrophysics, Masaryk University, Brno, Czech Republic \\ ${ }^{4}$ Center for Cosmology and Astrophysics, Alikhanian National Science Laboratory, Armenia
}

\begin{abstract}
To enlarge our database of Chemically Peculiar stars, we compiled published data concerning the Lambda Bootis stars observed by high-resolution spectroscopy techniques during last decades. In total, 62 Lambda Bootis stars have been added to the database. To deepen our knowledge on statistical overview of the abundance anomalies versus the physical parameters of stars, our data were compared with previous compilations, as well as with Gaia DR2 data. Different statistical tests were applied on our data for 20 chemical elements for the search of interesting correlations for different physical parameters in the atmospheres of those stars. We confirmed all previous results and obtained correlations between different physical parameters, but because of the lack of the data, we could not find new correlations. We hope, that Gaia EDR3 data, on which we are working now, will help us to improve our database and to understand the nature of all Chemically Peculiar stars.
\end{abstract}

Keywords: stars: abundances - stars: chemically peculiar - stars: $\lambda$ Bootis - methods: statistical techniques: spectroscopic - catalogue

\section{Introduction}

Statistical study of Chemically Peculiar stars we started during last year of my $\mathrm{PhD}$ thesis in 2012 with my supervisor from Paris Observatory/Meudon - Georges Alecian and continue to work on that project till nowadays. Later on, we enlarge our collaboration and include on it Artur Hakobyan (2018) - former BAO senior researcher and Ernst Paunzen (2020) from Masaryk University- an expert of $\lambda$ Bootis stars. During these years we published several articles and catalogues based on the statistical study of different type of Chemically Peculiar stars (Ghazaryan \& Alecian, 2016, Ghazaryan et al., 2018, 2019).

So, what we know about $\lambda$ Bootis stars? First $\lambda$ Bootis stars was discovered by Jesuit Father Angelo Secchi the Director of Vatican Observatory in 1860s', an excellent spectroscopist, who analysed more than 4000 stars spectroscopically and was very famous astronomer.

Later on, a few women- Morgan and Kellman in collaboration with Philip Kunan produced Henry Drapper Catalogue published between 1918-1924, giving spectroscopic classifications for more than 225.000 and in 1943 they published "An Atlas of Stellar Spectra" (Morgan et al., 1943). In fact, from that Atlas the study of $\lambda$ Bootis stars begins.

What Morgan and Kellman denote in "An Atlas of Stellar Spectra" about $\lambda$ Bootis stars, is that the spectral type of $\lambda$ Bootis stars in near A0, as far as can be determined. The spectral lines, while not unusually broad, are very weak, so that the only features easily visible are as weak $\mathrm{K}$ line and the Balmer series of hydrogen.

This idea was a key for Ernst Paunzen and Richard Gray to think that $\lambda$ Bootis stars may be peculiar ones and to think about the origin of that peculiarity.

At the end of twentieth century Gray showed that there is a so called "garbage bag" between Population I and Population II type stars and that bag is filled by $\lambda$ Bootis stars. In 1997 Gray showed an example of $\lambda$ Bootis star spectrum itself sandwiched in between the spectra of normal or standard stars (Gray, 1997). You can see a figure from Gray's paper (see Figure 1), where above the $\lambda$ Bootis star spectrum is HR 3314 star's spectrum and below $\beta$ Leo's spectrum. Effective temperature in the atmospheres of both stars is 


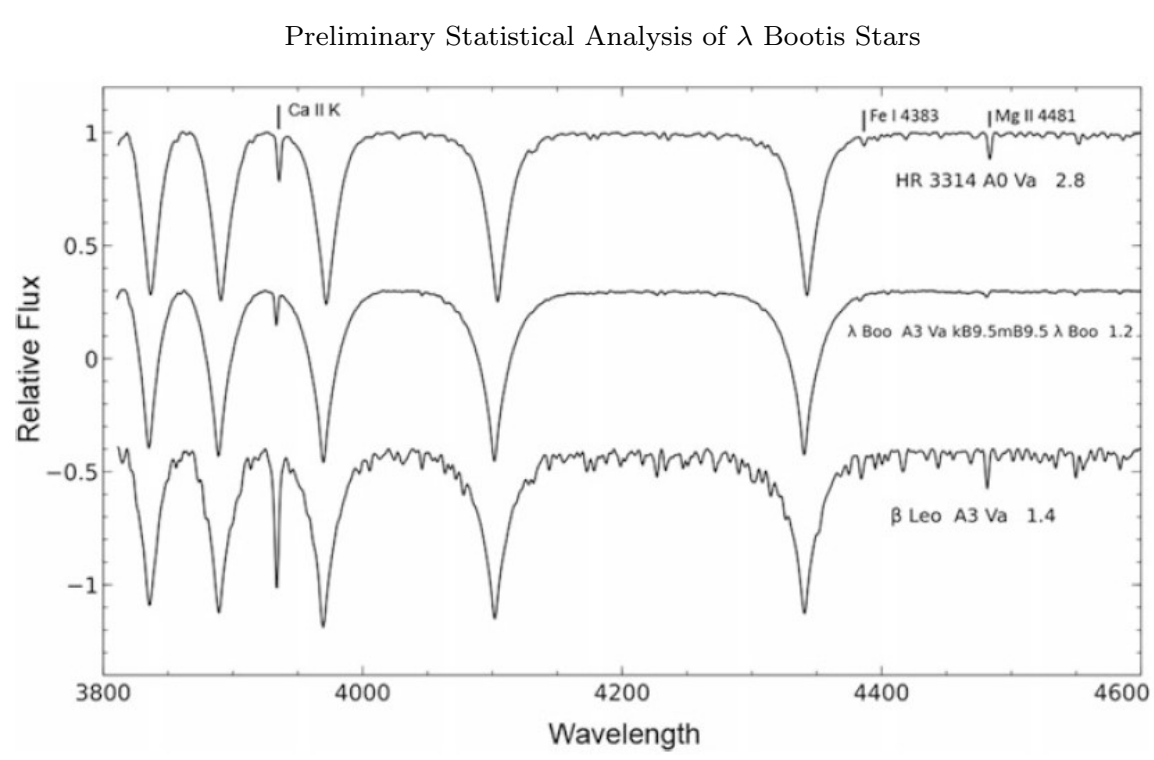

Figure 1. Montage of $\lambda$ Boo with an MK standard star above and below. The spectra are from the Dark Sky Observatory.

10,000 K. By comparison the spectral lines we see that for $\lambda$ Bootis stars they are broad, which means that those stars are fast rotators.

Bringed example of $\lambda$ Bootis star's spectrum shows that it is an A type star belonging to the $\mathrm{V}$ class fusing hydrogen to helium in its core, like the Sun. It can also be referred as a main sequence star. It has broad wings to it's hydrogen lines. "kB9.5" refers to the strength of the ionized calcium K line (CaII K) on the left side of the spectrum. Compare it to the substantial CaII K line in $\beta$ Leo, to which it should match given its' A3 temperature type, and to the still significant one in HR 3314. It is a bit weaker in $\lambda$ Boo even than A0, and if we took a look at late B-type stars, it would fit with a B9.5 spectrum. "mB9.5" is short for looking at the metal lines due to other elements, particularly iron. There are the tiny "wriggles" in the spectrum, which are weak like CaII K line, again having about the same strength matching a B9.5 star. A $\lambda$ Boo star is weak-lined, at least regarding to the strength of lines corresponding to its' temperature type, in this case A3. " $\lambda$ Boo" is the final judgement that this star doesn't just have weak metal lines, but that the weakness is itself peculiar and initiates the prototype, $\lambda$ Boo. A place to look for this is by comparing the ratio between the MgII $\lambda 4481$ line and the nearby FeI $\lambda 4383$ line. That ratio, which is equal to 1.2, appears at the end of the classification in Fig. 1 given by Gray in 1997. In $\lambda$ Boo the MgII line is noticeably weaker than the iron lines nearby, when compared with the spectrum corresponding to its metal lines, and that is the real clincher.

Unfortunately, even since the time of Gray's definition, not everyone was as careful as he is classifying a star as a $\lambda$ Bootis star and the "garbage bag" was filled with a lot of non-members of $\lambda$ Bootis stars group.

In 2015 Murphy published a catalogue of probably candidates of $\lambda$ Bootis stars, from which only 64 are proofed $\lambda$ Bootis stars, 103 being definitely non-members and the membership status for 45 of them is still unclear (Murphy et al., 2015).

If we have a look to the plot of chemical abundances of $\lambda$ Bootis stars given by Ernst Paunzen after Heiter in 2002 (see Figure 2), we see that four elements, which are shaded - carbon, nitrogen, oxygen and sulfur, compared to the standard stars abundances, have near normal abundances, whereas others can range to quite low values - especially for iron-peak elements (Heiter, 2002).

By summarizing the known facts about $\lambda$ Bootis stars, we can tell that they are A type stars, and the effective temperature in their atmospheres is found in the range of 7,500-9,000 K. There are strong underabundances of iron-peak elements in their atmospheres. Carbon, nitrogen, oxygen and sulfur are in the same amount as in the atmospheres of normal stars. They have high rotation velocities, small magnetic fields, and are found in the range of zero age mass stars.

\footnotetext{
*satenikghazarjan@yahoo.de, Corresponding author
} 


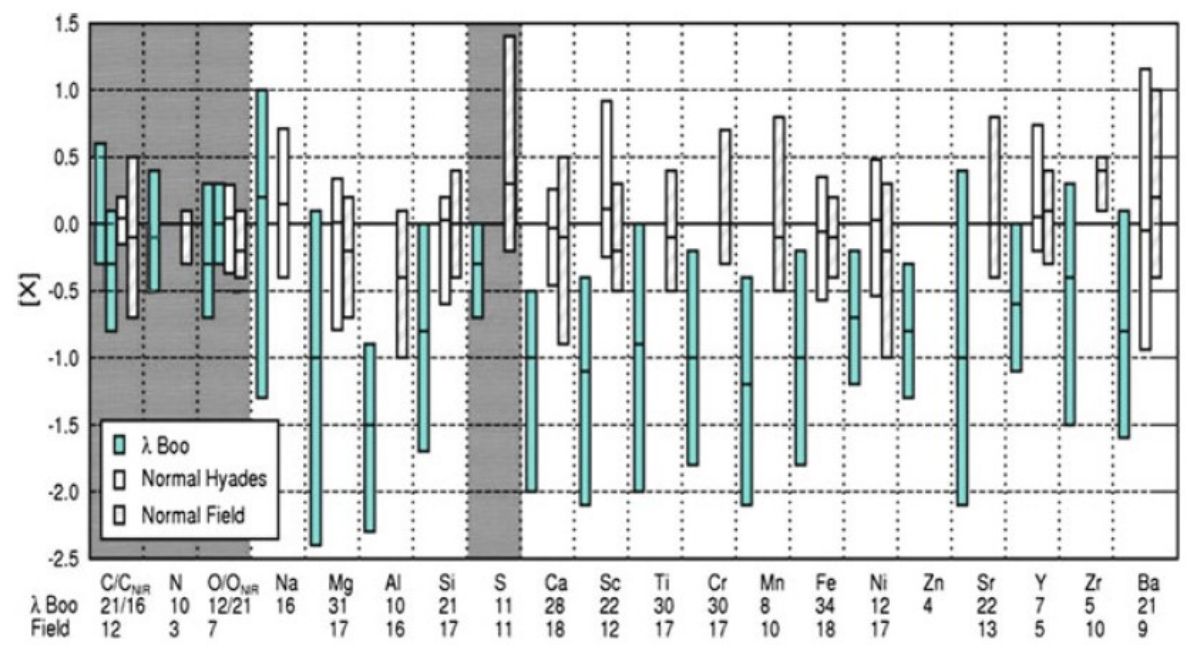

Figure 2. Abundance pattern for $\lambda$ Boo stars. Plot is created by Paunzen after Heiter (2002).

\section{Statistical study}

To do statistical analysis we combined from literature all $\lambda$ Bootis stars, which were observed by high resolution techniques. They were 65 in total. We combined their chemical abundances, known physical parameters - spectral type, effective temperature $\left(T_{\text {eff }}\right)$, gravity $(\log \mathrm{g})$, rotational velocity ( $\left.\mathrm{v} \sin i\right)$ and multiplicity from the papers from which their chemical abundances were taken, and did statistics on them. We applied Spearman Rank and Kendall $\tau$ correlation tests widely used on astronomy on chemical abundances, $T_{\text {eff }}, \log \mathrm{g}$ and on $\mathrm{v} \sin i$ of $\lambda$ Bootis stars to see the correlation between chemical abundances and physical parameters of those stars. To remind you what those tests are (Feigelson \& Babu, 2012), we should tell that Spearman's Rank correlation test performs a hypothesis test on pair of two variables with null hypothesis that they are independent, and alternative hypothesis that they are not. Usually, one accepts the alternative hypothesis of the test than p-value is less than 5 percent. We also pay attention to those cases when the p-values are close to the adopted threshold between 5 and 6 percent (marginal cases). Spearman's coefficient $\rho$ is a non-parametric measure of rank correlation $(\rho \in[-1 ; 1])$; it assesses how well the relationship between two variables can be described using a monotonic function. When each of the variables is a perfect monotonic function of the other, Spearman's coefficient $\rho$ is +1 or -1 .

Kendall $\tau$ test is also non-parametric test for statistical dependence based on the $\tau$ coefficient. It measures the rank correlation: the similarity of the orderings of the data when ranked by each of the quantities. The Kendall correlation between two variables will be high, when observations have a similar rank (correlation quantity is 1) between the two variables, and low, when observations have a dissimilar rank (correlation factor is -1) between the two variables.

The results, which we obtained, are plotted below (see Figure 3). We found

1) a strong correlation between carbon abundance and gravity,

2) an another strong correlation between oxygen abundance and rotational velocity,

3) a correlation between magnesium abundance and rotational velocity,

4) a correlation between silicon abundance and effective temperature,

5) a correlation between strontium abundance and gravity,

6) a correlation between yttrium abundance and gravity, and finally,

7) a correlation between zirconium abundance and effective temperature and also for gravity. 

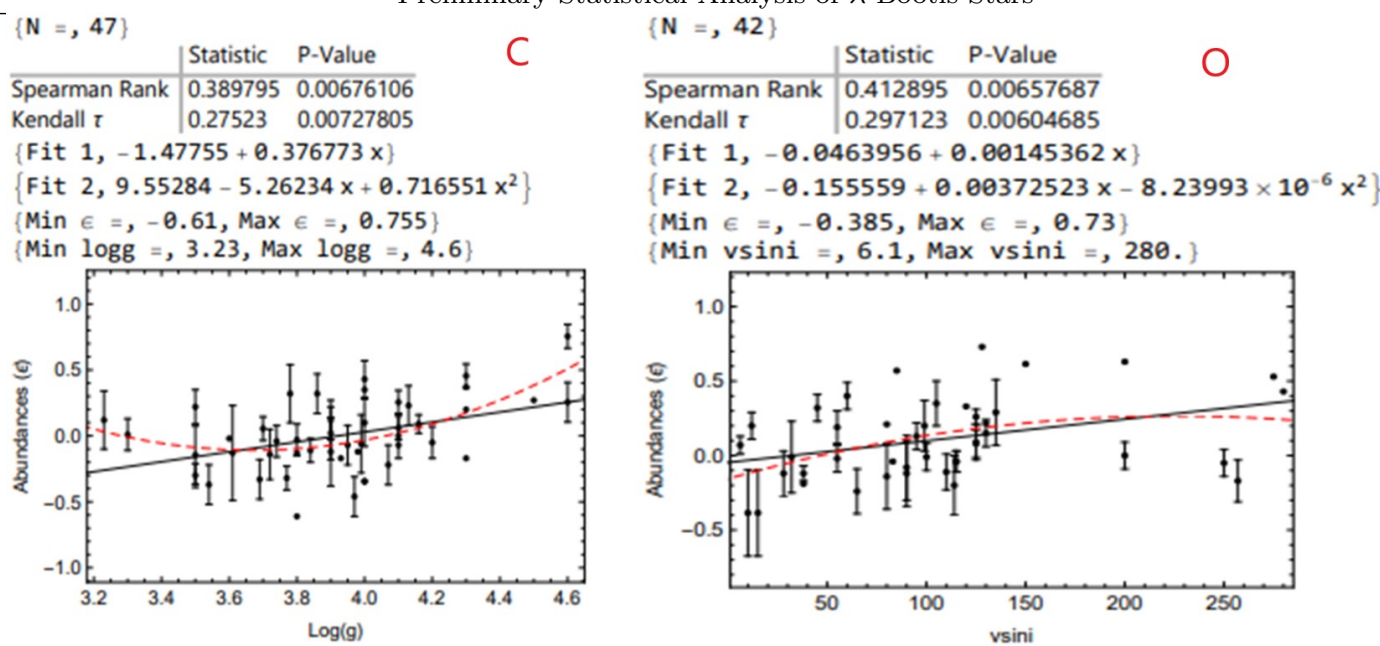

$\{\mathrm{N}=, 43\}$

$\{N=, 53\}$

\begin{tabular}{l|ll} 
& Statistic & P-Value \\
\hline Spearman Rank & 0.299256 & 0.0294941 \\
Kendall & 0.217905 & 0.0225823
\end{tabular}

\{Fit $1,-1.19818+0.00379384 x$ \}

Fit 2, $\left.-0.901461-0.00242856 x+0.0000227152 x^{2}\right\}$

Min $\epsilon=,-2.385, \operatorname{Max} \epsilon=, 0.3$ \}

(Min vsini $=, 6.1$, Max vsini $=, 300$.
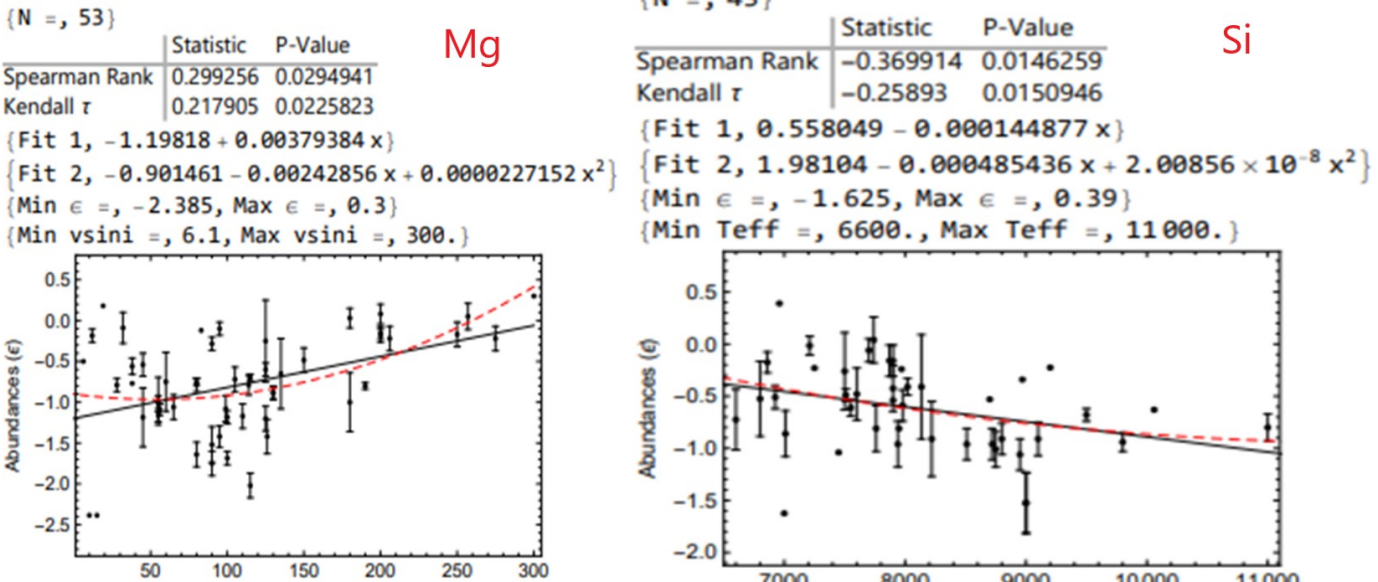

Min $\in=,-1.625$, Max $\in=0.39$

(Min Teff $=$, 6600., Max Teff $=, 11000$.
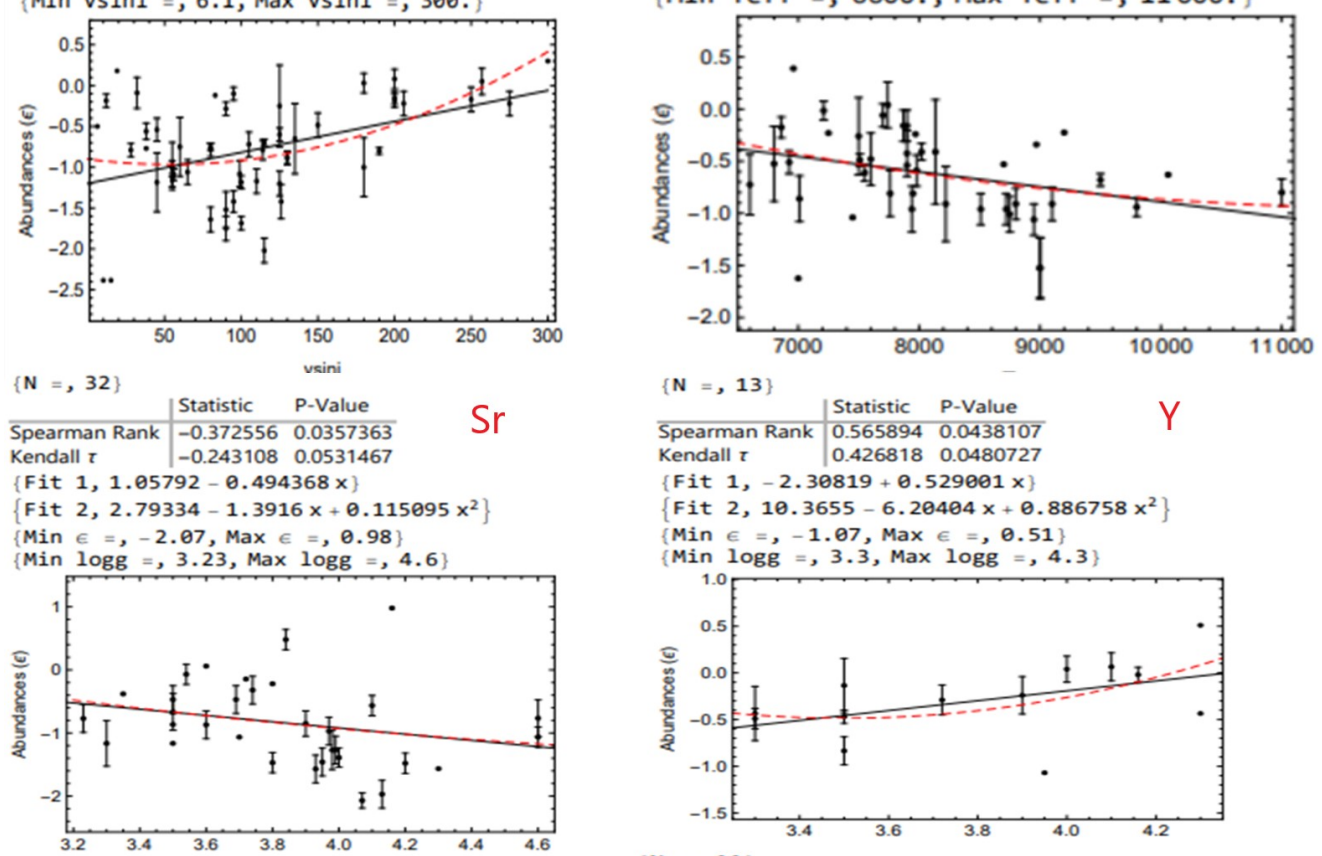

\begin{tabular}{l|lll}
$\{\mathrm{N}=, 13\}$ & & \\
& Statistic & P-Value & \\
\hline Spearman Rank & 0.565894 & 0.0438107 & $Y$
\end{tabular}

\begin{tabular}{l|lll} 
Kendall $\tau$ & 0.426818 & 0.0480727
\end{tabular}

\{Fit 1, $-2.30819+0.529001 \times$

Fit 2, 10.3655-6.20404x+0.886758 $\left.x^{2}\right\}$

Min $\epsilon=,-1.07, \operatorname{Max} \epsilon=, 0.51$

$\{$ Min $\operatorname{logg}=, 3.3$, Max logg $=, 4.3\}$

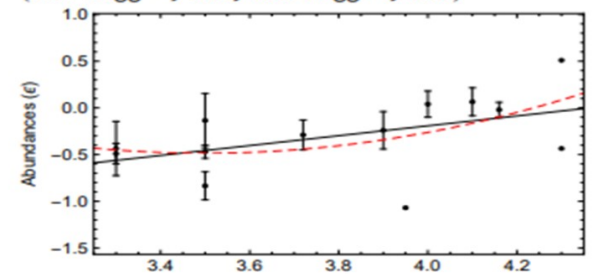

$\{\mathrm{N}=, 11\}$

\begin{tabular}{l|lll} 
& Statistic & P-Value & $\mathrm{Zr}$ \\
\hline Spearman Rank & 0.651482 & 0.0298838 \\
Kendall $\tau$ & 0.477084 & 0.0423302
\end{tabular}$\quad \mathrm{r}$

\{Fit $1,-3.05141+0.000359821 x$

\{Fit $2,-6.24977+0.00115202 \times-4.82626 \times 10^{-8} x^{2}$ \}

$\{$ Min $\in=,-1.445, \operatorname{Max} \in=, 0.44\}$

(Min Teff $=, 6800$., Max Teff $=, 9820$.
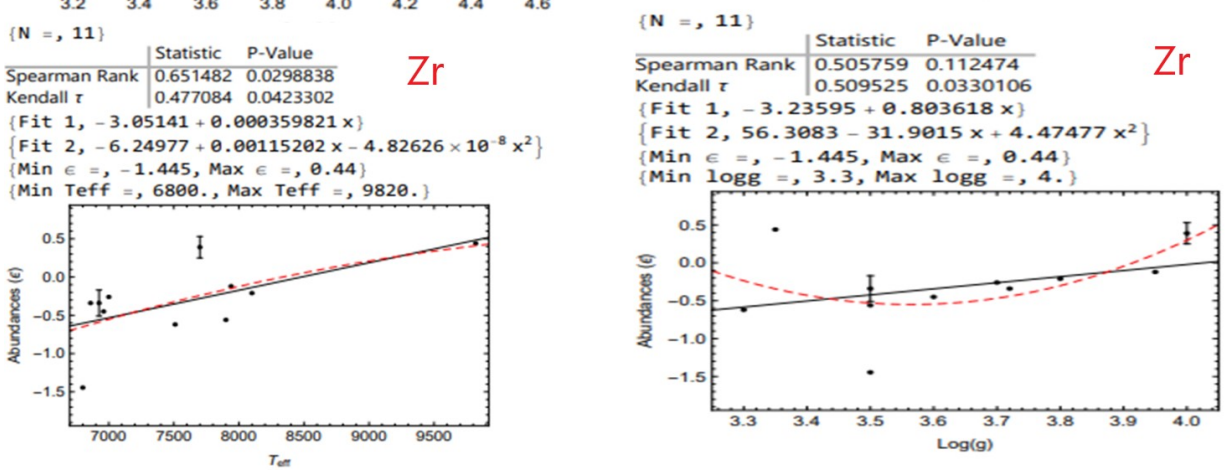

Figure 3. Correlations between $\lambda$ Boo stars chemical abundances and physical parameters.

\section{Conclusions}

As you can see from bringed plots, we have perfect correlations between different chemical abundances of $\lambda$ Bootis stars and their physical parameters. What we are going to do the next? It is, of course, to look to Gaia EDR3 data, which will help us to add much more confirmed $\lambda$ Boo stars to our database, including 
their physical parameters. In that case we can make our database homogenous and hopefully get better statistical results. They can help us to understand the nature of $\lambda$ Boo stars and summing up our whole research - the nature of all Chemically Peculiar stars.

\section{Acknowledgements}

SG would like to thank to all coauthors for fruitful discussions and collaboration.

\section{References}

Feigelson E. D., Babu G. J., 2012, Modern Statistical Methods for Astronomy

Ghazaryan S., Alecian G., 2016, Mon. Not. R. Astron. Soc. , 460, 1912

Ghazaryan S., Alecian G., Hakobyan A. A., 2018, Mon. Not. R. Astron. Soc. , 480, 2953

Ghazaryan S., Alecian G., Hakobyan A. A., 2019, Mon. Not. R. Astron. Soc. , 487, 5922

Gray R. O., 1997, in Philip A. G. D., Liebert J., Saffer R., Hayes D. S., eds, The Third Conference on Faint Blue Stars. p. 237

Heiter U., 2002, A\&A, 381, 959

Morgan W. W., Keenan P. C., Kellman E., 1943, An atlas of stellar spectra, with an outline of spectral classification

Murphy S. J., et al., 2015, PASA, 32, e036 